\title{
Analysis of a Retro-PN Guidance Law
}

\author{
Satadal Ghosh, Debasish Ghose and Soumyendu Raha
}

\begin{abstract}
This paper presents a complete analysis of a three dimensional retro-PN guidance law, suited for intercepting targets that are of higher speeds than the interceptors. It proposes a somewhat counter-intuitive guidance law that uses a negative navigation constant (as against the usual positive one) to show that this modification makes it possible to achieve collision conditions that were inaccessible to the standard law. An analysis for three dimensional engagements is presented in a modified polar coordinate system and complete capturability results are obtained. Simulation results are given to support the theoretical findings.
\end{abstract}

\section{INTRODUCTION}

Intercepting targets having speeds higher than the interceptor speed is a challenging task. There are very few papers in the literature that address this problem. Kuroda and Imado [1] showed that the near-head-on scenario is the best way for an interceptor to achieve small miss distances against a higher speed target. Golan and Shima [2] positioned the interceptor ahead of the higher speed target on its flight trajectory. The standard PN law, with positive navigation constant $N$, is a widely used guidance law, and has been analyzed extensively in the literature for 2-D engagements with lower speed targets [5], [6], [7]. A 3-D version was discussed by Tyan [3] with the aid of a modified polar coordinate system, which was later extended to higher speed targets by Tyan and Shen [4].

Prasanna and Ghose [8], [9], [10] showed that for intercepting a higher speed target, there exists two collision conditions (as against only one for a lower speed target), and one of them can be achieved with positive $N$ (standard PN) while the other can be achieved by using a negative $N$ (retro-PN). However, all these studies were confined to a 2-D space. Since the 3D engagements are more practical, this paper analyzes the capturability of retro-PN guidance law for 3-D engagement scenarios, using a modified polar co-ordinate system given in [3], [4], [11]. Fig. 1(a) gives an idea about the perspective in which this paper makes a contribution.

Satadal Ghosh is a PhD student in IISc Mathematics Initiative and the Guidance, Control, and Decision Systems Laboratory in the Department of Aerospace Engineering, Indian Institute of Science, Bangalore, India. satadaldaero.iisc.ernet. in

Debasish Ghose is a professor in the Guidance, Control, and Decision Systems Laboratory in the Department of Aerospace Engineering, Indian Institute of Science, Bangalore, India. dghose@aero.iisc.ernet.in

Soumyendu Raha is an associate professor in the Supercomputer Education and Research Centre, Indian Institute of Science, Bangalore, India rahalserc.iisc.ernet.in

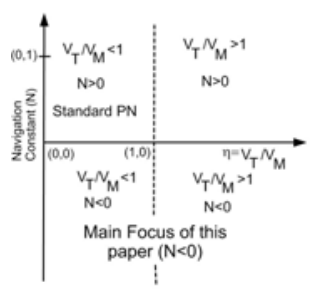

(a) Focus of present work

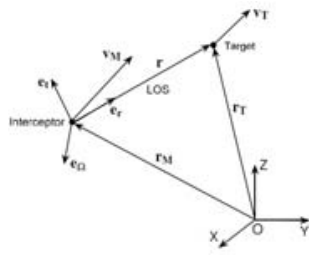

(b) Engagement Geometry
Fig. 1. Focus of present work and Basic engagement geometry

\section{Preliminaries and Problem Formulation}

\section{A. Preliminaries}

Consider the engagement geometry shown in Fig. 1(b). In the line-of-sight (LOS) fixed reference frame [4], [3], the relative position vector $\mathbf{r}$ (LOS vector), relative velocity vector $\dot{\mathbf{r}}$ and relative acceleration vector $\ddot{\mathbf{r}}$ between the target and the interceptor are defined as below.

$$
\begin{aligned}
& \mathbf{r} \triangleq \rho \mathbf{e}_{\mathbf{r}} \triangleq \mathbf{r}_{\mathbf{T}}-\mathbf{r}_{\mathbf{M}} \\
& \dot{\mathbf{r}}=\dot{\rho} \mathbf{e}_{\mathbf{r}}+\rho \dot{\mathbf{e}}_{\mathbf{r}}=\dot{\rho} \mathbf{e}_{\mathbf{r}}+\rho\left(\Omega \times \mathbf{e}_{\mathbf{r}}\right)=\mathbf{v}_{\mathbf{T}}-\mathbf{v}_{\mathbf{M}} \\
& \ddot{\mathbf{r}}=\ddot{\rho} \mathbf{e}_{\mathbf{r}}+2 \dot{\rho} \dot{\mathbf{e}}_{\mathbf{r}}+\rho \ddot{\mathbf{e}}_{\mathbf{r}}=\mathbf{a}_{\mathbf{T}}-\mathbf{a}_{\mathbf{M}}
\end{aligned}
$$

$\mathbf{e}_{\mathbf{r}}$ is the unit vector along the LOS and $\dot{\mathbf{e}}_{\mathbf{r}}=\Omega \times \mathbf{e}_{\mathbf{r}}$ and the angular velocity of the LOS, $\Omega$ is orthogonal to the LOS.

$$
\Omega=\rho^{-1} \mathbf{e}_{\mathbf{r}} \times\left(\mathbf{v}_{\mathbf{T}}-\mathbf{v}_{\mathbf{M}}\right)
$$

Magnitude of the angular velocity of LOS vector with sign

$$
\omega \triangleq\left\{\begin{array}{c}
\|\Omega\|, \text { when } \mathbf{e}_{\mathbf{r}} \text { moves anticlockwise } \\
-\|\Omega\|, \text { when } \mathbf{e}_{\mathbf{r}} \text { moves clockwise }
\end{array}\right.
$$

Define $\mathbf{e}_{\mathbf{t}} \triangleq \dot{\mathbf{e}}_{\mathbf{r}} / \omega, \quad \mathbf{e}_{\Omega} \triangleq \Omega / \omega$. Clearly $\mathbf{e}_{\mathbf{r}} \times \mathbf{e}_{\mathbf{t}}=\mathbf{e}_{\Omega}$. $\mathbf{e}_{\mathbf{r}}, \mathbf{e}_{\mathbf{t}}$ and $\mathbf{e}_{\Omega}$ are the orthogonal unit vectors of the modified LOSfixed polar co-ordinate system.

\section{B. Retro-PN Guidance Law}

The latax command for the retro-PN guidance law is:

$\mathbf{a}_{\mathbf{M}}=-\left(-\beta V_{M} \mathbf{e}_{\mathbf{M}} \times \Omega\right)=\beta V_{M} \omega\left[\left(\mathbf{e}_{\mathbf{M}}^{\prime} \mathbf{e}_{\mathbf{t}}\right) \mathbf{e}_{\mathbf{r}}-\left(\mathbf{e}_{\mathbf{M}}{ }^{\prime} \mathbf{e}_{\mathbf{r}}\right) \mathbf{e}_{\mathbf{t}}\right]$

where, $-\beta$ is the navigation constant, with $\beta>0, V_{M}$ is the speed of the interceptor and $\mathbf{e}_{\mathbf{M}}$ is the unit vector along the interceptor velocity vector. Note that in standard PN guidance law, the navigation constant is always positive.

\section{Dynamics of relative velocity and direction cosines}

Define $u \triangleq \dot{\rho}, v \triangleq \rho \omega, w \triangleq \rho^{-1}$. Here $u$ and $v$ denote the relative velocity of the target with respect to the interceptor 
along the LOS and along the transverse direction $\mathbf{e}_{\mathbf{t}}$ respectively and $w$ is the inverse of the range.

$$
u=V_{T}\left(\mathbf{e}_{\mathbf{T}}^{\prime} \mathbf{e}_{\mathbf{r}}\right)-V_{M}\left(\mathbf{e}_{\mathbf{M}}^{\prime} \mathbf{e}_{\mathbf{r}}\right) ; v=V_{T}\left(\mathbf{e}_{\mathbf{T}}^{\prime} \mathbf{e}_{\mathbf{t}}\right)-V_{M}\left(\mathbf{e}_{\mathbf{M}}{ }^{\prime} \mathbf{e}_{\mathbf{t}}\right)
$$

where, $\mathbf{e}_{\mathbf{T}}$ is the unit vector along the target velocity vector. For a non-maneuvering target, $\mathbf{a}_{\mathbf{T}}=0$. Transforming the independent variable from $t$ to $\tau$ by $d \tau \triangleq w d t$, we obtain,

$$
\begin{aligned}
& d u / d \tau=\left[v-\beta V_{M}\left(\mathbf{e}_{\mathbf{M}}^{\prime} \mathbf{e}_{\mathbf{t}}\right)\right] v, u\left(\tau_{0}\right)=u_{0} \\
& d v / d \tau=\left[-u+\beta V_{M}\left(\mathbf{e}_{\mathbf{M}}^{\prime} \mathbf{e}_{\mathbf{r}}\right)\right] v, v\left(\tau_{0}\right)=v_{0} \\
& d w / d \tau=-u w, w\left(\tau_{0}\right)=w_{0} .
\end{aligned}
$$

$v=0$ is the equilibrium in the $(u, v)$-plane. The differential equations related to the direction cosines associated with $\mathbf{e}_{\mathbf{M}}$ with respect to $\theta$ are obtained as below, where $d \theta \triangleq \nu d \tau$.

$$
\begin{aligned}
& d\left(\mathbf{e}_{\mathbf{M}}^{\prime} \mathbf{e}_{\mathbf{r}}\right) / d \boldsymbol{\theta}=(\beta+1) \mathbf{e}_{\mathbf{M}}^{\prime} \mathbf{e}_{\mathbf{t}}, \mathbf{e}_{\mathbf{M}}^{\prime} \mathbf{e}_{\mathbf{r}}\left(\theta_{0}\right)=\left(\mathbf{e}_{\mathbf{M}}^{\prime} \mathbf{e}_{\mathbf{r}}\right)_{0} \\
& d\left(\mathbf{e}_{\mathbf{M}}^{\prime} \mathbf{e}_{\mathbf{t}}\right) / d \boldsymbol{\theta}=-(\beta+1) \mathbf{e}_{\mathbf{M}}^{\prime} \mathbf{e}_{\mathbf{r}}, \mathbf{e}_{\mathbf{M}}^{\prime} \mathbf{e}_{\mathbf{t}}\left(\theta_{0}\right)=\left(\mathbf{e}_{\mathbf{M}} \mathbf{e}_{\mathbf{t}}\right)_{0} \\
& d\left(\mathbf{e}_{\mathbf{M}}^{\prime} \mathbf{e}_{\Omega}\right) / d \boldsymbol{\theta}=0, \mathbf{e}_{\mathbf{M}}^{\prime} \mathbf{e}_{\Omega}\left(\theta_{0}\right)=\left(\mathbf{e}_{\mathbf{M}}^{\prime} \mathbf{e}_{\Omega}\right)_{0}
\end{aligned}
$$

Solving (11) and (12), direction cosines of $\mathbf{e}_{\mathbf{M}}$ are obtained,

$$
\left[\begin{array}{l}
\mathbf{e}_{\mathbf{M}}^{\prime} \mathbf{e}_{\mathbf{r}}(\boldsymbol{\theta}) \\
\mathbf{e}_{\mathbf{M}}^{\prime} \mathbf{e}_{\mathbf{t}}(\boldsymbol{\theta})
\end{array}\right]=R\left[-(\beta+1)\left(\boldsymbol{\theta}-\theta_{0}\right)\right]\left[\begin{array}{c}
\left(\mathbf{e}_{\mathbf{M}}^{\prime} \mathbf{e}_{\mathbf{r}}\right)_{0} \\
\left(\mathbf{e}_{\mathbf{M}}^{\prime} \mathbf{e}_{\mathbf{t}}\right)_{0}
\end{array}\right]
$$

where, $R[]=.\left[\begin{array}{cc}\cos (.) & \sin (.) \\ -\sin (.) & \cos (.)\end{array}\right]$.

The dynamic equations related to $u$ and $v$ in terms of $\theta$ are,

$$
\begin{aligned}
& {\left[\begin{array}{l}
d u / d \theta \\
d v / d \theta
\end{array}\right]=\left[\begin{array}{cc}
0 & 1 \\
-1 & 0
\end{array}\right]\left[\begin{array}{l}
u \\
v
\end{array}\right]+} \\
& \beta V_{M}\left[\begin{array}{cc}
0 & -1 \\
1 & 0
\end{array}\right] R\left[-(\beta+1)\left(\theta-\theta_{0}\right)\right]\left[\begin{array}{c}
\left(\mathbf{e}_{\mathbf{M}}^{\prime} \mathbf{e}_{\mathbf{r}}\right)_{0} \\
\left(\mathbf{e}_{\mathbf{M}}^{\prime} \mathbf{e}_{\mathbf{t}}\right)_{0}
\end{array}\right]
\end{aligned}
$$

Solving (15), $u$ and $v$ are obtained as,

$$
\begin{array}{r}
{\left[\begin{array}{l}
u(\theta) \\
v(\theta)
\end{array}\right]=R\left[-\left(\boldsymbol{\theta}-\theta_{0}\right)\right]\left[\begin{array}{c}
u_{0}+V_{M}\left(\mathbf{e}_{\mathbf{M}}^{\prime} \mathbf{e}_{\mathbf{r}}\right)_{0} \\
v_{0}+V_{M}\left(\mathbf{e}_{\mathbf{M}}^{\prime} \mathbf{e}_{\mathbf{t}}\right)_{0}
\end{array}\right]} \\
-R\left[-(\beta+1)\left(\boldsymbol{\theta}-\theta_{0}\right)\right]\left[\begin{array}{c}
V_{M}\left(\mathbf{e}_{\mathbf{M}}^{\prime} \mathbf{e}_{\mathbf{r}}\right)_{0} \\
V_{M}\left(\mathbf{e}_{\mathbf{M}}^{\prime} \mathbf{e}_{\mathbf{t}}\right)_{0}
\end{array}\right]
\end{array}
$$

Using (7), it follows that,

$$
\begin{gathered}
{\left[\begin{array}{l}
u(\boldsymbol{\theta}) \\
v(\boldsymbol{\theta})
\end{array}\right]=R\left[-\left(\boldsymbol{\theta}-\theta_{0}\right)\right]\left[\begin{array}{c}
V_{T}\left(\mathbf{e}_{\mathbf{T}}^{\prime} \mathbf{e}_{\mathbf{r}}\right)_{0} \\
V_{T}\left(\mathbf{e}_{\mathbf{T}}^{\prime} \mathbf{e}_{\mathbf{t}}\right)_{0}
\end{array}\right]} \\
-R\left[-(\beta+1)\left(\boldsymbol{\theta}-\theta_{0}\right)\right]\left[\begin{array}{c}
V_{M}\left(\mathbf{e}_{\mathbf{M}}^{\prime} \mathbf{e}_{\mathbf{r}}\right)_{0} \\
V_{M}\left(\mathbf{e}_{\mathbf{M}}^{\prime} \mathbf{e}_{\mathbf{t}}\right)_{0}
\end{array}\right]
\end{gathered}
$$

Therefore, direction cosines for $\mathbf{e}_{\mathbf{T}}$ can be written as,

$$
\left[\begin{array}{c}
\mathbf{e}_{\mathbf{T}}{ }^{\prime} \mathbf{e}_{\mathbf{r}}(\boldsymbol{\theta}) \\
\mathbf{e}_{\mathbf{T}} \mathbf{e}_{\mathbf{t}}(\boldsymbol{\theta})
\end{array}\right]=R\left[-\left(\boldsymbol{\theta}-\theta_{0}\right)\right]\left[\begin{array}{c}
\left(\mathbf{e}_{\mathbf{T}}^{\prime} \mathbf{e}_{\mathbf{r}}\right)_{0} \\
\left(\mathbf{e}_{\mathbf{T}}^{\prime} \mathbf{e}_{\mathbf{t}}\right)_{0}
\end{array}\right]
$$

\section{Trajectories in the normalized $(u, v)$-plane}

The trajectory of the engagement in the normalized $(u, v)$ plane is obtained as a moving cycloid with fixed radius and varying centre. Let $\eta \triangleq V_{T} / V_{M}, \bar{u} \triangleq u / V_{T}, \bar{v} \triangleq v / V_{T}$. Note that $\mathbf{v}_{\mathbf{T}}-\mathbf{v}_{\mathbf{M}}$ has no component in $\mathbf{e}_{\Omega}$ direction.

$$
V_{T}\left(\mathbf{e}_{\mathbf{T}}^{\prime} \mathbf{e}_{\Omega}\right)=V_{M}\left(\mathbf{e}_{\mathbf{M}}^{\prime} \mathbf{e}_{\Omega}\right) \Rightarrow\left(\mathbf{e}_{\mathbf{T}}^{\prime} \mathbf{e}_{\Omega}\right)=\eta^{-1}\left(\mathbf{e}_{\mathbf{M}}^{\prime} \mathbf{e}_{\Omega}\right)
$$

By (13), $V_{M}\left(\mathbf{e}_{\mathbf{M}}{ }^{\prime} \mathbf{e}_{\Omega}\right)=V_{M}\left(\mathbf{e}_{\mathbf{M}} \mathbf{e}_{\Omega}\right)_{0}$ and since $\mathbf{a}_{\mathbf{T}}=0$, $V_{T}\left(\mathbf{e}_{\mathbf{T}}{ }^{\prime} \mathbf{e}_{\Omega}\right)=V_{T}\left(\mathbf{e}_{\mathbf{T}}{ }^{\prime} \mathbf{e}_{\Omega}\right)_{0}$. From (7), the trajectories in the $(\bar{u}, \bar{v})$-plane are given in two alternative forms below.

$$
\begin{aligned}
& {\left[\bar{u}+\eta^{-1}\left(\mathbf{e}_{\mathbf{M}}{ }^{\prime} \mathbf{e}_{\mathbf{r}}\right)\right]^{2}+\left[\bar{v}+\eta^{-1}\left(\mathbf{e}_{\mathbf{M}}{ }^{\prime} \mathbf{e}_{\mathbf{t}}\right)\right]^{2}=1-\eta^{-2}\left(\mathbf{e}_{\mathbf{M}}{ }^{\prime} \mathbf{e}_{\Omega}\right)_{0}^{2}} \\
& {\left[\bar{u}-\left(\mathbf{e}_{\mathbf{T}}{ }^{\prime} \mathbf{e}_{\mathbf{r}}\right)\right]^{2}+\left[\bar{v}-\left(\mathbf{e}_{\mathbf{T}}{ }^{\prime} \mathbf{e}_{\mathbf{t}}\right)\right]^{2}=\eta^{-2}-\left(\mathbf{e}_{\mathbf{T}}{ }^{\prime} \mathbf{e}_{\Omega}\right)_{0}^{2}}
\end{aligned}
$$

For the feasibility of trajectories given by (20) and (21) the conditions are $\left(\mathbf{e}_{\mathbf{M}}{ }^{\prime} \mathbf{e}_{\Omega}\right)_{0}^{2}<\eta^{2} ; \quad\left(\mathbf{e}_{\mathbf{T}}{ }^{\prime} \mathbf{e}_{\Omega}\right)_{0}^{2}<\eta^{-2}$ respectively.

\section{E. Feasible final conditions}

For successful interception, the conditions at the final instant are given by $\bar{v}_{f}=0$ and $\bar{u}_{f}<0$ [12]. The set of initial conditions that would lead to successful interception by the retro-PN guided interceptor, that is, the above-mentioned feasible final conditions, are called the capture zone of the retro-PN guidance law. Solving (20) and (21) separately with constraint $\bar{v}_{f}=0$, we obtain (22) and (23) respectively.

$$
\begin{aligned}
& \bar{u}_{f}=-\eta^{-1}\left(\mathbf{e}_{\mathbf{M}}^{\prime} \mathbf{e}_{\mathbf{r}}\right)_{f} \pm \eta^{-1} \sqrt{\left(\mathbf{e}_{\mathbf{M}}^{\prime} \mathbf{e}_{\mathbf{r}}\right)_{f}^{2}+\left(\eta^{2}-1\right)} \\
& \bar{u}_{f}=\left(\mathbf{e}_{\mathbf{T}}^{\prime} \mathbf{e}_{\mathbf{r}}\right)_{f} \pm \sqrt{\left(\mathbf{e}_{\mathbf{T}} \mathbf{e}_{\mathbf{r}}\right)_{f}^{2}-\left(1-\eta^{-2}\right)}
\end{aligned}
$$

From (22), if $\eta<1$, then for $\bar{u}_{f}<0,\left(\mathbf{e}_{\mathbf{M}}{ }^{\prime} \mathbf{e}_{\mathbf{r}}\right)_{f}>0$ and $\sqrt{1-\eta^{2}} \leq\left(\mathbf{e}_{\mathbf{M}}{ }^{\prime} \mathbf{e}_{\mathbf{r}}\right)_{f} \leq 1$. If $\eta>1$, then for $\bar{u}_{f}<0,\left(\mathbf{e}_{\mathbf{M}}{ }^{\prime} \mathbf{e}_{\mathbf{r}}\right)_{f}$ can be either positive or negative or zero, but the interception is possible only for one set of initial conditions for which (22) holds with '-' sign. From (23), if $\eta<1$, then at $\bar{u}_{f}<0$, $\left(\mathbf{e}_{\mathbf{T}}{ }^{\prime} \mathbf{e}_{\mathbf{r}}\right)_{f}$ can be either positive or negative or zero, but the interception would be possible only for one set of initial conditions for which (23) holds with '-' sign. If $\eta>1$, then at $\bar{u}_{f}<0,\left(\mathbf{e}_{\mathbf{T}}^{\prime} \mathbf{e}_{\mathbf{r}}\right)_{f}<0$ and $-1 \leq\left(\mathbf{e}_{\mathbf{T}}^{\prime} \mathbf{e}_{\mathbf{r}}\right)_{f} \leq-\sqrt{\left(1-\eta^{-2}\right)}$.

\section{CAPturability AnAlysis}

A. Some preliminary results

$$
\begin{aligned}
& x \triangleq \sqrt{\left(\mathbf{e}_{\mathbf{M}} \mathbf{e}_{\mathbf{r}}\right)_{0}^{2}+\left(\mathbf{e}_{\mathbf{M}} \mathbf{e}_{\mathbf{t}}\right)_{0}^{2}}=\sqrt{1-\left(\mathbf{e}_{\mathbf{M}} \mathbf{e}_{\Omega}\right)_{0}^{2}} \\
& y \triangleq \sqrt{\left(\mathbf{e}_{\mathbf{T}} \mathbf{e}_{\mathbf{r}}\right)_{0}^{2}+\left(\mathbf{e}_{\mathbf{T}} \mathbf{e}_{\mathbf{t}}\right)_{0}^{2}}=\sqrt{1-\left(\mathbf{e}_{\mathbf{T}} \mathbf{e}_{\Omega}\right)_{0}^{2}}
\end{aligned}
$$

Clearly, $0 \leq x, y \leq 1$.

Lemma 1: $\eta>1 \Rightarrow x \leq y, \eta<1 \Rightarrow x \geq y . \eta=1 \Rightarrow x=y$.

Proof: From (19) and subsequent discussions, we get,

$$
\begin{aligned}
& 1-x^{2}=\eta^{2}\left(1-y^{2}\right) \Rightarrow y^{2}=\left(x \eta^{-1}\right)^{2}+\left(1-\eta^{-2}\right) \\
\Rightarrow & \left(1-x^{2}\right) /\left(1-y^{2}\right)=\eta^{2}
\end{aligned}
$$

For $x=y=1, \eta$ can be any number greater than or equal to or less than 1. Hence from (26), (27), the lemma follows.

Since $\mathbf{e}_{\mathbf{M}}{ }^{\prime} \mathbf{e}_{\Omega}$ and $\mathbf{e}_{\mathbf{T}}{ }^{\prime} \mathbf{e}_{\Omega}$ remain constant throughout the mission time, $x$ and $y$ would also remain same throughout the engagement time. Define $\cos \theta_{M_{0}} \triangleq\left(\mathbf{e}_{\mathbf{M}}{ }^{\prime} \mathbf{e}_{\mathbf{r}}\right)_{0} / x, \sin \theta_{M_{0}} \triangleq$ $\left(\mathbf{e}_{\mathbf{M}}{ }^{\prime} \mathbf{e}_{\mathbf{t}}\right)_{0} / x ; \cos \theta_{T_{0}} \triangleq\left(\mathbf{e}_{\mathbf{T}}{ }^{\prime} \mathbf{e}_{\mathbf{r}}\right)_{0} / y, \sin \theta_{T_{0}} \triangleq\left(\mathbf{e}_{\mathbf{T}}{ }^{\prime} \mathbf{e}_{\mathbf{t}}\right)_{0} / y$. Therefore, referring to (17), $\bar{u}$ and $\bar{v}$ can be written as,

$$
\begin{gathered}
\bar{u}=y \cos \left(\theta_{T_{0}}-\triangle \theta\right)-(x / \eta) \cos \left(\theta_{M_{0}}-(\beta+1) \triangle \theta\right) \\
\bar{v}=y \sin \left(\theta_{T_{0}}-\triangle \theta\right)-(x / \eta) \sin \left(\theta_{M_{0}}-(\beta+1) \triangle \theta\right)
\end{gathered}
$$




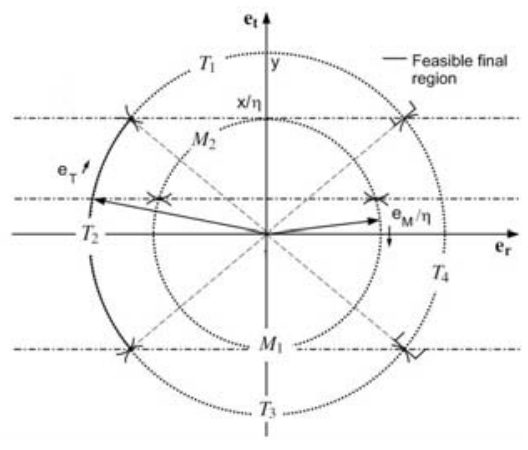

Fig. 2. $\left(\mathbf{e}_{\mathbf{r}}, \mathbf{e}_{\mathbf{t}}\right)$-plane and feasible region for $\bar{v}_{f}=0$

where, $\triangle \theta \triangleq \theta-\theta_{0}$. Hence, in the $\left(\mathbf{e}_{\mathbf{r}}, \mathbf{e}_{\mathbf{t}}\right)$-plane the projections of the two vectors $\mathbf{e}_{\mathbf{T}}$ and $\mathbf{e}_{\mathbf{M}} / \eta$ can be thought of as rotating in two circles centered at origin with radius $y$ and $x / \eta$ respectively. For $\beta>0$, if $v>0$, then they rotate in the clockwise direction and if $v<0$, then they rotate in the anticlockwise direction. The projection of $\mathbf{e}_{\mathbf{M}} / \eta$ rotates $(\beta+1)$ times faster than that of $\mathbf{e}_{\mathbf{T}}$. Hence $\bar{u}$ can be considered as the difference of the projections of $\mathbf{e}_{\mathbf{T}}$ and $\mathbf{e}_{\mathbf{M}} / \eta$ along the LOS, that is in the $\mathbf{e}_{\mathbf{r}}$ direction and $\bar{v}$ as the difference of their components in $\mathbf{e}_{\mathbf{t}}$ direction.

The combinations of $v_{0}$ and $u_{0}$ (or the combinations of $v_{0}, \theta_{T_{0}}$ and $\left.\theta_{M_{0}}\right)$, which lead to the final conditions $\bar{v}_{f}=0$ and $\bar{u}_{f}<0$, define the capture zone. The combinations of $v_{0}$, $\theta_{T_{0}}$ and $\theta_{M_{0}}$, for which $\bar{v}_{0}=0$ and $\bar{u}_{0}<0$, define the trivial capture zone, since when $\bar{v}_{0}=0$, the system is already at equilibrium and the negative relative velocity along the LOS leads to successful interception. The capture zone, other than the trivial capture zone, would be referred to as the nontrivial capture zone. Define $z \triangleq(x / \eta y)$.

Lemma 2: If $\eta>1$, then there exists a feasible nontrivial capturability region in terms of $\theta_{M_{0}}$ and $\theta_{T_{0}}$.

Proof: For successful engagement, the conditions at the final instant are $\bar{v}_{f}=0$ and $\bar{u}_{f}<0$. By Lemma $1, \eta>1 \Rightarrow$ $x<y$ and hence, $z=x / \eta y<1$. Then with respect to $\theta_{T}$, the $\left(\mathbf{e}_{\mathbf{r}}, \mathbf{e}_{\mathbf{t}}\right)$-plane can be segmented into five disjoint regions as shown in Fig.2. $T_{1}=\left\{\theta_{T} \mid \sin ^{-1} z \leq \theta_{T}<\pi-\sin ^{-1} z\right\}$; $T_{2}=\left\{\theta_{T} \mid \pi-\sin ^{-1} z<\theta_{T}<\pi+\sin ^{-1} z\right\} ; T_{3}=\left\{\theta_{T} \mid \pi+\right.$ $\left.\sin ^{-1} z<\theta_{T}<2 \pi-\sin ^{-1} z\right\} ; T_{4}=\left\{\theta_{T} \mid \theta_{T} \in\left[0, \sin ^{-1} z\right) \cup\right.$ $\left.\left[2 \pi-\sin ^{-1} z, 2 \pi\right)\right\} ; T_{5}=\left\{\pi-\sin ^{-1} z, \pi+\sin ^{-1} z\right\}$.

All these regions are defined modulo $2 \pi$. For any $\theta_{T}$, three disjoint regions $M_{1}=\left\{\theta_{M} \mid v>0\right\}, M_{2}=\left\{\theta_{M} \mid v<0\right\}, M_{3}$ $=\left\{\theta_{M} \mid v=0\right\}$ are defined. Clearly $\theta_{T_{0}} \in T_{1} \Rightarrow v_{0}>0$ and $\theta_{T_{0}} \in T_{3} \Rightarrow v_{0}<0$ for any $\theta_{M_{0}} . \bar{v}_{f}=0$ is only possible at $\theta_{T_{f}} \in T_{2} \cup T_{4} \cup T_{5}$. From (28) and (29), we have,

$$
\left[(\bar{u} / y)-\cos \left(\theta_{T_{0}}-\triangle \theta\right)\right]^{2}+\left[\bar{v} / y-\sin \left(\theta_{T_{0}}-\triangle \theta\right)\right]^{2}=z^{2}
$$

As $z^{2}<1, \bar{u}_{f}<0$ if $\pi / 2<\theta_{T_{0}}-\triangle \theta_{f}<3 \pi / 2$. For $\bar{v}_{f}=0$,

$$
\bar{u}_{f} / y=\cos \left(\theta_{T_{0}}-\triangle \theta_{f}\right) \pm \sqrt{z^{2}-\sin ^{2}\left(\theta_{T_{0}}-\triangle \theta_{f}\right)}
$$

From (31), $\left|\sin \left(\theta_{T_{0}}-\triangle \theta_{f}\right)\right|<z$. Therefore, the conditions of successful interception $\bar{v}_{f}=0$ and $\bar{u}_{f}<0$ are possible only if $\theta_{T_{f}} \in T_{2} \cup T_{5}$. By the intermediate value theorem, clearly, given any real $\beta$, the normalized relative velocity in the transverse direction $\bar{v}$, being a smooth function of $\Delta \theta$, as given in (29), must cross zero before changing its sign.

In Scenario 1, since $v_{0}>0$, the projection of both $\mathbf{e}_{\mathbf{T}}$ and $\mathbf{e}_{\mathbf{M}} / \eta$ rotate in the $\left(\mathbf{e}_{\mathbf{r}}, \mathbf{e}_{\mathbf{t}}\right)$-plane in the clockwise direction. $\bar{v}$ has to be zero before the projection of $\mathbf{e}_{\mathbf{T}}$ reaches the $T_{3}$ region of $\theta_{T}$, as in this region $\bar{v}<0$, that is, $\bar{v}$ would become zero in the $T_{4}$ region of $\theta_{T}$, where, $\bar{u}_{f}>0$. Hence, this case would not lead to any feasible interception. In Scenario 5, $v_{0}<0$ and hence the projection of both $\mathbf{e}_{\mathbf{T}}$ and $\mathbf{e}_{\mathbf{M}} / \eta$ rotate in the $\left(\mathbf{e}_{\mathbf{r}}, \mathbf{e}_{\mathbf{t}}\right)$-plane in the anticlockwise direction. $\bar{v}$ has to be zero before $\mathbf{e}_{\mathbf{T}}$ reaches the $T_{1}$ region of $\theta_{T}$, as in this region $\bar{v}>0$, that is, $\bar{v}$ would become zero in $T_{2}$ or $T_{4}$ or $T_{5}$ region of $\theta_{T}$. By selection of proper proportional navigation gain $\beta$, this $\bar{v}$ can be made equal to zero in $T_{2}$ or $T_{5}$ region of $\theta_{T}$, where, $\bar{u}_{f}<0$ leading towards feasible interception.

By similar logic for all scenarios in the disjoint and exhaustive set of initial conditions, mentioned in Table I, if $\eta>1$, then $\theta_{T_{0}} \in T_{2}$ in combination with $\theta_{M_{0}} \in\left(M_{1} \cup\right.$ $M_{2}$ ) form nontrivial capture zone, while $\theta_{T_{0}} \in T_{2} \cup T_{5}$ in combination with $\theta_{M_{0}} \in M_{3}$ form trivial capture zone.

However note that for feasible interception in any scenario, $\left(\mathbf{e}_{\mathbf{M}}{ }^{\prime} \mathbf{e}_{\mathbf{r}}\right)_{f} \leq 0$, that is, for $\bar{v}_{0}>0, \theta_{M_{f}}=\pi / 2+\delta$, where, $\delta \in C$ and for $\bar{v}_{0}<0, \theta_{M_{f}}=3 \pi / 2-\delta$, where, $\delta \in D$, where $C$ and $D$ are defined in Lemma 5 and 6 respectively. Following similar arguments, the following results can be proved.

Lemma 3: If $\eta=1$, then there exists a feasible nontrivial capturability region when $\theta_{T_{0}} \in T_{2}$ and $\theta_{M_{0}} \in[0,2 \pi)$.

Lemma 4: If $\eta<1$, then there does not exist any nontrivial feasible capturability region in terms of $\theta_{T_{0}}$ and $\theta_{M_{0}}$.

From Lemma 2, 3 and 4, it is evident that a retro-PN guided interceptor would have a feasible nontrivial capture zone if and only if $\eta \geq 1$, provided $\beta>0$ in (6). Now the existence of such $\beta>0$ would be analyzed in the following two lemma for $\bar{v}_{0}>0$ and $\bar{v}_{0}<0$ respectively.

Lemma 5: If $\eta \geq 1, \theta_{T_{0}} \in T_{2}$ and $\bar{v}_{0}>0$, then

1) If $\theta_{M_{0}} \in Q_{1}=[0, \pi / 2)$, then $\forall \delta \in C, \exists \beta>0 \ni$ $3 \pi / 2+\theta_{M_{0}}-\delta=(\beta+1)\left(\theta_{T_{0}}-\pi+\sin ^{-1}(z \cos \delta)\right)$.

2) If $\theta_{M_{0}} \in Q_{2} \cup Q_{3}$, where $Q_{2}=(3 \pi / 2,2 \pi)$ and $Q_{3}=$ $[\pi / 2,3 \pi / 2]$, then $\forall \delta \in C, \exists \beta>0 \ni$ $\theta_{M_{0}}-\pi / 2-\delta=(\beta+1)\left(\theta_{T_{0}}-\pi+\sin ^{-1}(z \cos \delta)\right)$. where, $C=\left[0, \cos ^{-1}\left(z^{-1} \sin \left(\pi-\theta_{T_{0}}\right)\right)\right)$.

Proof: $\quad T_{2} a=\left\{\theta_{T} \mid \pi-\sin ^{-1} z<\theta_{T} \leq \pi\right\}, \quad T_{2} b=$ $\left\{\theta_{T} \mid \pi<\theta_{T}<\pi+\sin ^{-1} z\right\}$ and $T_{2}=T_{2} a \cup T_{2} b$. When $\theta_{T_{0}} \in T_{2} a$, then $\bar{v}_{0}>0 \Leftrightarrow \theta_{M_{0}} \in M_{1} a \cup M_{1} b$, where $M_{1} a=$ $\left[0, \sin ^{-1}\left(z^{-1} \sin \theta_{T_{0}}\right)\right), M_{1} b=\left(\pi-\sin ^{-1}\left(z^{-1} \sin \theta_{T_{0}}\right), 2 \pi\right)$. When $\theta_{T_{0}} \in T_{2} b$, then $\bar{v}_{0}>0 \Leftrightarrow \theta_{M_{0}} \in M_{1} b$.

Case 1: $\left(\theta_{T_{0}} \in T_{2} a, \theta_{M_{0}} \in M_{1} a \subseteq Q_{1}\right)$ Since $\delta<\cos ^{-1}\left(z^{-1} \sin \left(\pi-\theta_{T_{0}}\right)\right) \leq \pi / 2$ and $\theta_{M_{0}} \geq 0$, $3 \pi / 2+\theta_{M_{0}}-\delta>3 \pi / 2-\cos ^{-1}\left(z^{-1} \sin \left(\pi-\theta_{T_{0}}\right)\right) \geq \pi$. But, since $\pi-\sin ^{-1} z<\theta_{T_{0}} \leq \pi$ and $\delta \geq 0, \theta_{T_{0}}-\pi+$ $\sin ^{-1}(z \cos \delta) \leq \theta_{T_{0}}-\pi+\sin ^{-1}(z)<\pi$. Hence, if $\theta_{T_{0}} \in$ $T_{2} a, \theta_{M_{0}} \in M_{1} a$, then $\forall \delta \in C$ and for some $\beta>0$,

$$
\begin{aligned}
& 3 \pi / 2+\theta_{M_{0}}-\delta>\theta_{T_{0}}-\pi+\sin ^{-1}(z \cos \delta) \\
\Leftrightarrow & 3 \pi / 2+\theta_{M_{0}}-\delta=(\beta+1)\left(\theta_{T_{0}}-\pi+\sin ^{-1}(z \cos \delta)\right)
\end{aligned}
$$


TABLE I

PRESENCE OF NONTRIVIAL CAPTURE ZONE FOR $x<\eta y$

\begin{tabular}{|c|c|c|c|c|c|c|}
\hline $\begin{array}{c}\text { Scenario } \\
\text { No. }\end{array}$ & $\begin{array}{c}\text { Combination of } \\
\theta_{T_{0}} \text { and } \theta_{M_{0}}\end{array}$ & $\begin{array}{c}\text { Rotation of } \\
\mathbf{e}_{\mathbf{T}} \text { and } \mathbf{e}_{\mathbf{M}} / \eta\end{array}$ & $\begin{array}{c}\bar{v}_{f}=0 \\
\text { at } \theta_{T_{f}} \in\end{array}$ & $\bar{u}_{f}$ & $\begin{array}{c}\text { Feasible } \\
\text { Interception }\end{array}$ & $\begin{array}{c}\text { Nontrivial } \\
\text { Capture Zone }\end{array}$ \\
\hline 1 & $\left\{T_{1} \cup T_{4}\right\}$ and $\left\{M_{1}\right\}$ & clockwise & $\left\{T_{4}\right\}$ & $>0$ & No & No \\
\hline 2 & $\left\{T_{3} \cup T_{4}\right\}$ and $\left\{M_{2}\right\}$ & anticlockwise & $\left\{T_{4}\right\}$ & $>0$ & No & No \\
\hline 3 & $\left\{T_{4}\right\}$ and $\left\{M_{3}\right\}$ & none & $\left\{T_{4}\right\}$ & $>0$ & No & No \\
\hline 4 & $\left\{T_{2}\right\}$ and $\left\{M_{1}\right\}$ & clockwise & $\left\{T_{2} \cup T_{5}\right\}$ & $<0$ & Yes & Yes \\
\hline 5 & $\left\{T_{2}\right\}$ and $\left\{M_{2}\right\}$ & anticlockwise & $\left\{T_{2} \cup T_{5}\right\}$ & $<0$ & Yes & Yes \\
\hline 6 & $\left\{T_{2}\right\}$ and $\left\{M_{3}\right\}$ & none & $\left\{T_{2}\right\}$ & $<0$ & Yes & No \\
\hline 7 & $\left\{T_{5}\right\}$ and $\left\{M_{1}\right\}$ & clockwise & $\left\{T_{4}\right\}$ & $>0$ & No & No \\
\hline 8 & $\left\{T_{5}\right\}$ and $\left\{M_{2}\right\}$ & anticlockwise & $\left\{T_{4}\right\}$ & $>0$ & No & No \\
\hline 9 & $\left\{T_{5}\right\}$ and $\left\{M_{3}\right\}$ & none & $\left\{T_{5}\right\}$ & $<0$ & Yes & No \\
\hline
\end{tabular}

Case 2: $\left(\theta_{T_{0}} \in T_{2} a \cup T_{2} b, \theta_{M_{0}} \in M_{1} b \subseteq Q_{2} \cup Q_{3}\right)$ Since $\theta_{M_{0}} \in M_{1} b, \theta_{M_{0}}>\pi-\sin ^{-1}\left(z^{-1} \sin \theta_{T_{0}}\right)$. Here the function $\delta+\sin ^{-1}(z \cos \delta)$ is increasing in $\delta$ over $C$, since $x \leq \eta y$. Therefore, for any given $\theta_{T_{0}} \in T_{2}$, we have, $\delta+\sin ^{-1}(z \cos \delta)<\cos ^{-1}\left(z^{-1} \sin \left(\pi-\theta_{T_{0}}\right)\right)+\pi-\theta_{T_{0}}$. Thus, $\theta_{M_{0}}-\pi / 2-\delta-\theta_{T_{0}}+\pi-\sin ^{-1}(z \cos \delta)>0$. So if $\theta_{T_{0}} \in$ $T_{2} a \cup T_{2} b, \theta_{M_{0}} \in M_{1} b$, then $\forall \delta \in C$ and for some $\beta>0$,

$$
\begin{aligned}
& \theta_{M_{0}}-\pi / 2-\delta>\theta_{T_{0}}-\pi+\sin ^{-1}(z \cos \delta) \\
\Leftrightarrow & \theta_{M_{0}}-\pi / 2-\delta=(\beta+1)\left(\theta_{T_{0}}-\pi+\sin ^{-1}(z \cos \delta)\right)
\end{aligned}
$$

By similar logic the next result for $\bar{v}_{0}<0$ can be proved. Lemma 6: If $\eta \geq 1, \theta_{T_{0}} \in T_{2}$ and $\bar{v}_{0}<0$, then

1) If $\theta_{M_{0}} \in Q_{1} \cup Q_{3}$, then $\forall \delta \in D, \exists \beta>0 \ni$ $3 \pi / 2-\theta_{M_{0}}-\delta=(\beta+1)\left(\pi+\sin ^{-1}(z \cos \delta)-\theta_{T_{0}}\right)$.

2) If $\theta_{M_{0}} \in Q_{2}$, then $\forall \delta \in D, \exists \beta>0 \ni$ $7 \pi / 2-\theta_{M_{0}}-\delta=(\beta+1)\left(\pi+\sin ^{-1}(z \cos \delta)-\theta_{T_{0}}\right)$.

where, $D=\left[0, \pi-\cos ^{-1}\left(z^{-1} \sin \left(\pi-\theta_{T_{0}}\right)\right)\right)$ and $Q_{1}, Q_{2}, Q_{3}$ are as defined in Lemma 5.

\section{B. Some definitions}

Given $\theta_{T_{0}} \in T_{2}$, for $\bar{v}_{0}<0$ also, two regions of $\theta_{M_{0}}$, namely $M_{2} a=\left(\max \left(0, \sin ^{-1}\left(z^{-1} \sin \theta_{T_{0}}\right)\right), \pi-\right.$ $\left.\sin ^{-1}\left(z^{-1} \sin \theta_{T_{0}}\right)\right)$ and specifically for $\theta_{T_{0}} \in T_{2} b, M_{2} b=$ $\left(2 \pi+\sin ^{-1}\left(z^{-1} \sin \theta_{T_{0}}\right), 2 \pi\right)$ are defined.

Now for $\eta \geq 1$, define the following regions.

$R_{1}=\left\{\bar{v}_{0}, \theta_{T_{0}}, \theta_{M_{0}} \mid \bar{v}_{0}>0, \theta_{T_{0}} \in T_{2}, \theta_{M_{0}} \in M_{1} b\right\}$

$R_{2}=\left\{\bar{v}_{0}, \theta_{T_{0}}, \theta_{M_{0}} \mid \bar{v}_{0}>0, \theta_{T_{0}} \in T_{2} a, \theta_{M_{0}} \in M_{1} a\right\}$

$R_{3}=\left\{\bar{v}_{0}, \theta_{T_{0}}, \theta_{M_{0}} \mid \bar{v}_{0}<0, \theta_{T_{0}} \in T_{2}, \theta_{M_{0}} \in M_{2} a\right\}$

$R_{4}=\left\{\bar{v}_{0}, \theta_{T_{0}}, \theta_{M_{0}} \mid \bar{v}_{0}<0, \theta_{T_{0}} \in T_{2} b, \theta_{M_{0}} \in M_{2} b\right\}$

$R_{5}=\left\{\bar{v}_{0}, \theta_{T_{0}}, \theta_{M_{0}} \mid \bar{v}_{0}=0, \theta_{T_{0}} \in T_{2}\right\}$.

Clearly, $R_{5}$ forms the trivial capture zone. To analyze the nontrivial capture zone define the following set.

$$
C R_{R P N}=R_{1} \cup R_{2} \cup R_{3} \cup R_{4}
$$

Define $f_{1}, f_{2}: C \rightarrow \mathbb{R}^{+}$and $f_{3}, f_{4}: D \rightarrow \mathbb{R}^{+}$as $f_{1}(\delta)=\left(\theta_{M_{0}}-\pi / 2-\delta\right) /\left(\theta_{T_{0}}-\pi+\sin ^{-1}(z \cos \delta)\right)-1$, $f_{2}(\boldsymbol{\delta})=\left(3 \pi / 2+\theta_{M_{0}}-\delta\right) /\left(\theta_{T_{0}}-\pi+\sin ^{-1}(z \cos \delta)\right)-1$, $f_{3}(\delta)=\left(3 \pi / 2-\theta_{M_{0}}-\delta\right) /\left(\pi+\sin ^{-1}(z \cos \delta)-\theta_{T_{0}}\right)-1$, $f_{4}(\delta)=\left(7 \pi / 2-\theta_{M_{0}}-\delta\right) /\left(\pi+\sin ^{-1}(z \cos \delta)-\theta_{T_{0}}\right)-1$, which are convex functions in their respective domains of $\delta$, where, $C$ and $D$ are as mentioned in Lemma 5 and 6 respectively. Therefore, over $C$, both $f_{1}(\delta)$ and $f_{2}(\delta)$ and over $D$, both $f_{3}(\delta)$ and $f_{4}(\delta)$ have unique minima at $\underline{\delta}_{1}$, $\underline{\delta}_{2}, \underline{\delta}_{3}$ and $\underline{\delta}_{4}$ respectively and are positive as ensured by Lemma 5 and 6 respectively. $\beta_{i}=\left(f_{i}\left(\underline{\boldsymbol{\delta}}_{i}\right)\right) \forall i=1,2,3,4$.

\section{Capturability analysis without LOS turn rate constraint}

Theorem 1: Consider a Retro-PN guided ideal interceptor, pursuing a non-maneuvering target with $\eta=V_{T} / V_{M} \geq 1$. The necessary and sufficient condition for the existence of nontrivial capture zone with no constraints on LOS turn rate are $\left(\bar{v}_{0}, \theta_{T_{0}}, \theta_{M_{0}}\right) \in C R_{R P N}$, where, $C R_{R P N}$ is given by (32) and (1) if $\left(\bar{v}_{0}, \theta_{T_{0}}, \theta_{M_{0}}\right) \in R_{1}, \beta \geq \underline{\beta}_{1}$; (2) if $\left(\bar{v}_{0}, \theta_{T_{0}}, \theta_{M_{0}}\right) \in$ $R_{2}, \beta \geq \underline{\beta}_{2}$; (3) if $\left(\bar{v}_{0}, \theta_{T_{0}}, \theta_{M_{0}}\right) \in R_{3}, \beta \geq \underline{\beta}_{3}$ and (4) if $\left(\bar{v}_{0}, \theta_{T_{0}}, \theta_{M_{0}}\right) \in R_{4}, \beta \geq \underline{\beta}_{4}$.

Proof: From Lemma 2 , if $\eta \geq 1$, the nontrivial capture zone exists if and only if $\theta_{T_{0}} \in T_{2}$ and $\bar{v}_{0} \gtrless 0$. The set $S=\left\{\bar{v}_{0}, \theta_{T_{0}}, \theta_{M_{0}} \mid \bar{v}_{0} \gtrless 0, \theta_{T_{0}} \in T_{2}\right\}$ can be segmented into four mutually disjoint and exhaustive subsets $R_{1}, R_{2}, R_{3}, R_{4}$, while by (32), $R_{1} \cup R_{2} \cup R_{3} \cup R_{4}=C R_{R P N}=S$. Therefore, $\left(\bar{v}_{0}, \theta_{T_{0}}, \theta_{M_{0}}\right) \in C R_{R P N}$ forms one of the necessary and sufficient conditions for existence of nontrivial capture zone.

Consider $\left(\bar{v}_{0}, \theta_{T_{0}}, \theta_{M_{0}}\right) \in R_{1}$. Note in Fig. 3 that given a $\theta_{T_{0}} \in T_{2}$, for feasible interception, $\bar{v}_{f}$ can be zero for any $\delta \in$ $\left[0, \cos ^{-1}\left(z^{-1} \sin \left(\pi-\theta_{T_{0}}\right)\right)\right)=C$. For the proof of necessity of $\beta \geq \underline{\beta}_{1}$, consider that $\bar{v}_{f}=0$ for some $\delta=\delta^{\prime} \in C$. Therefore, the angle of rotation of the projections of $\mathbf{e}_{\mathbf{T}}$ and $\mathbf{e}_{\mathbf{M}} / \eta$ in the $\left(\mathbf{e}_{\mathbf{r}}, \mathbf{e}_{\mathbf{t}}\right)$-plane can be related as below.

$$
\begin{aligned}
& \left(\theta_{M_{0}}-\pi / 2-\delta^{\prime}\right) /(\beta+1)=\theta_{T_{0}}-\pi+\sin ^{-1}\left(z \cos \delta^{\prime}\right) \\
\Rightarrow & \beta^{\prime}=\left(\theta_{M_{0}}-\pi / 2-\delta^{\prime}\right) /\left(\theta_{T_{0}}-\pi+\sin ^{-1}\left(z \cos \delta^{\prime}\right)\right)-1
\end{aligned}
$$

From Lemma $5, \beta^{\prime}>0$ exists and by the definition of $\underline{\delta}_{1}$ and $\underline{\beta}_{1}$, we can conclude that $\beta^{\prime} \geq \underline{\beta}_{1}$. For the proof of sufficiency of $\beta \geq \beta_{1}$, we need to show that $\exists \delta \in C \ni$ $f_{1}(\delta)=\beta$. By definition of $\underline{\beta}_{1}$, for $\beta=\underline{\beta}_{1}$, it is trivially true. Now consider some $\beta=\beta^{\prime} \ni \beta^{\prime}>\underline{\beta}_{1} \cdot f_{1}(\delta) \rightarrow \infty$ as $\delta \rightarrow \cos ^{-1}\left(z^{-1} \sin \left(\pi-\theta_{T_{0}}\right)\right)$. By the intermediate value theorem, $f_{1}(\delta)$, being continuous over the domain $C$, takes all values in the range $\left[\beta_{1}, \infty\right)$. Therefore, $\exists \delta=\delta^{\prime} \in C \ni$ for $\beta=\beta^{\prime}>\underline{\beta}_{1}, \beta^{\prime}=f_{1}\left(\delta^{\prime}\right)$. Hence, $\beta \geq \underline{\beta}_{1}$ is both necessary and sufficient for the existence of the nontrivial capture zone if $\left(\bar{v}_{0}, \theta_{T_{0}}, \theta_{M_{0}}\right) \in R_{1}$.

By similar analysis of $f_{2}: C \rightarrow \mathbb{R}^{+}, f_{3}: D \rightarrow \mathbb{R}^{+}$and $f_{4}$ : 


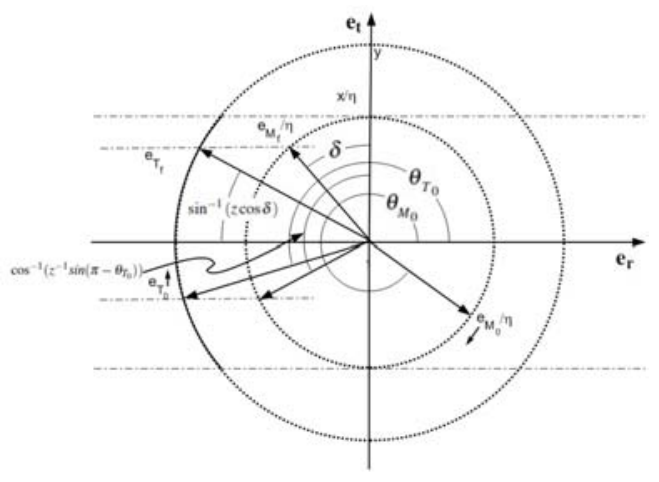

Fig. 3. Rotation of the projections of $\mathbf{e}_{\mathbf{T}}$ and $\mathbf{e}_{\mathbf{M}} / \eta$ in $\left(\mathbf{e}_{\mathbf{r}}, \mathbf{e}_{\mathbf{t}}\right)$-plane

$D \rightarrow \mathbb{R}^{+}$in case of $\left(\bar{v}_{0}, \theta_{T_{0}}, \theta_{M_{0}}\right) \in R_{2},\left(\bar{v}_{0}, \theta_{T_{0}}, \theta_{M_{0}}\right) \in R_{3}$ and $\left(\bar{v}_{0}, \theta_{T_{0}}, \theta_{M_{0}}\right) \in R_{4}$ respectively; we obtain that $\beta \geq \beta_{2}$, $\beta \geq \underline{\beta}_{3}$ and $\beta \geq \underline{\beta}_{4}$ are necessary and sufficient conditions for existence of nontrivial capture zone in respective cases.

Finally, as it has already been proved that $\left(\bar{v}_{0}, \theta_{T_{0}}, \theta_{M_{0}}\right) \in$ $R_{1} \cup R_{2} \cup R_{3} \cup R_{4}=C R_{R P N}=S$ forms a part of the necessary and sufficient conditions of successful interception.

\section{Capturability analysis with constraint on LOS turn rate}

Lemma 7: If $\left(\bar{v}_{0}, \theta_{T_{0}}, \theta_{M_{0}}\right) \in R_{1}$, then for $F_{1}(\delta)=$ $\left(f_{1}(\delta)+2\right)^{2}$ and if $\left(\bar{v}_{0}, \theta_{T_{0}}, \theta_{M_{0}}\right) \in R_{2}$, then for $F_{2}(\delta)=$ $\left(f_{2}(\delta)+2\right)^{2}$, both defined over $C$, there exists at least one interval of $\delta$ in $C^{-}=\left(0, \cos ^{-1}\left(z^{-1} \sin \left(\pi-\theta_{T_{0}}\right)\right)\right)$, at which $F_{1}(\delta)$ and $F_{2}(\delta)$ are respectively greater than $g_{1}(\delta)=4(1+$ $\left.\left(\eta^{2}-1\right) /\left(x^{2} \cos ^{2}(\pi / 2+\delta)\right)\right)$, defined over $C^{-}$.

Proof: If $\left(\bar{v}_{0}, \theta_{T_{0}}, \theta_{M_{0}}\right) \in R_{1} \cup R_{2}$, then $f_{1}(\delta)$ and $f_{2}(\delta)$, as well as $F_{1}(\delta)$ and $F_{2}(\delta)$, are defined over the domain $C=\left[0, \cos ^{-1}\left(z^{-1} \sin \left(\pi-\theta_{T_{0}}\right)\right)\right)$. By construction, $F_{1}(\delta)$ and $F_{2}(\delta)$ are continuous convex functions over $C$ and they attain their unique minimum at $\delta=\underline{\delta}_{1}$ and $\delta=\underline{\delta}_{2}$ respectively.

As $\delta \rightarrow \cos ^{-1}\left(z^{-1} \sin \left(\pi-\theta_{T_{0}}\right)\right), F_{1}(\delta), F_{2}(\delta) \rightarrow \infty$. Hence, by the intermediate value theorem, $F_{1}(\delta) \in\left[F_{1}\left(\underline{\delta}_{1}\right), \infty\right)$ and $F_{2}(\delta) \in\left[F_{2}\left(\underline{\delta}_{2}\right), \infty\right)$. As $\delta \rightarrow 0, g_{1}(\delta) \rightarrow \infty$ and as $\delta$ increases, $g_{1}(\delta)$ assumes finite positive values and finally as $\delta \rightarrow \cos ^{-1}\left(z^{-1} \sin \left(\pi-\theta_{T_{0}}\right)\right), g_{1}(\delta) \rightarrow g_{1}\left(\cos ^{-1}\left(z^{-1} \sin (\pi-\right.\right.$ $\left.\left.\theta_{T_{0}}\right)\right)$ ), which is positive finite. Therefore, there exists at least one $\delta \in C^{-}$such that $F_{1}(\delta)=g_{1}(\delta)$. Similar argument holds for $F_{2}(\delta)$ and $g_{1}(\delta)$. Since $g_{1}(\delta)$ is convex over its domain $C^{-}$, it can intersect $F_{1}(\delta)$ a finite number of times in $C^{-} . \mathbb{D}_{1} \triangleq\left\{\delta \in C^{-} \mid g_{1}(\delta)=F_{1}(\delta)\right\}, \mathbb{D}_{2} \triangleq\{\delta \in$ $\left.C^{-} \mid g_{1}(\delta)=F_{2}(\delta)\right\}$ are nonempty finite cardinality sets of positive elements and hence have their maximals. Let $\tilde{\delta}_{11} \triangleq \max \left\{\mathbb{D}_{1}\right\}, \tilde{\delta}_{21} \triangleq \max \left\{\mathbb{D}_{2}\right\}, \quad \tilde{\delta}_{12}=\tilde{\delta}_{22} \triangleq \sup \left\{C^{-}\right\}=$ $\cos ^{-1}\left(z^{-1} \sin \left(\pi-\theta_{T_{0}}\right)\right)$.

$F_{1}^{\prime}(\delta) \triangleq d F_{1}(\delta) / d \delta, \quad F_{2}^{\prime}(\delta) \triangleq d F_{2}(\delta) / d \delta, \quad g_{1}^{\prime}(\delta) \triangleq$ $d g_{1}(\delta) / d \delta$. By the smoothness of $F_{1}^{\prime}(\delta)$ and $F_{2}^{\prime}(\delta)$ over $C^{-}$, clearly $\underline{\delta}_{1}, \underline{\delta}_{2}<\pi / 2$. Therefore, as $\delta \rightarrow \tilde{\delta}_{12}=\tilde{\delta}_{22}$, $F_{1}^{\prime}(\delta), F_{2}^{\prime}(\delta)>0$. If $\tilde{\delta}_{12}=\tilde{\delta}_{22} \leq \pi / 2$, then $g_{1}^{\prime}(\delta)<0$ as $\delta \rightarrow \tilde{\delta}_{12}=\tilde{\delta}_{22}$, in which case trivially $F_{1}(\delta)>g_{1}(\delta), \forall \delta \in$ $\left(\tilde{\delta}_{11}, \tilde{\delta}_{12}\right)$ and $F_{2}(\delta)>g_{1}(\delta) \forall \delta \in\left(\tilde{\delta}_{21}, \tilde{\delta}_{22}\right)$. Otherwise, $g_{1}^{\prime}(\boldsymbol{\delta})>0 ; F_{1}(\delta), F_{2}(\delta) \rightarrow \infty ; F_{1}^{\prime}(\delta) / g_{1}^{\prime}(\delta), F_{2}^{\prime}(\delta) / g_{1}^{\prime}(\delta) \rightarrow$

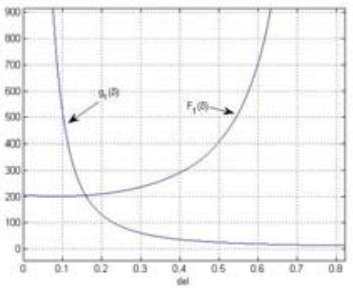

Fig. 4. $\quad F_{1}(\delta)$ and $g_{1}(\delta)$

$\infty$ as $\delta \rightarrow \tilde{\delta}_{12}=\tilde{\delta}_{22}$, hence, $F_{1}(\delta)>g_{1}(\delta) \forall \delta \in\left(\tilde{\delta}_{11}, \tilde{\delta}_{12}\right)$ and $F_{2}(\delta)>g_{1}(\delta) \forall \delta \in\left(\tilde{\delta}_{21}, \tilde{\delta}_{22}\right)$.

Lemma 8: If $\left(\bar{v}_{0}, \theta_{T_{0}}, \theta_{M_{0}}\right) \in R_{3}$, then for $F_{3}(\delta)=$ $\left(f_{3}(\delta)+2\right)^{2}$ and if $\left(\bar{v}_{0}, \theta_{T_{0}}, \theta_{M_{0}}\right) \in R_{4}$, then for $F_{4}(\delta)=$ $\left(f_{4}(\delta)+2\right)^{2}$, both defined over $D$, there exists at least one interval of $\delta$ in $D^{-}=\left(0, \pi-\cos ^{-1}\left(z^{-1} \sin \left(\pi-\theta_{T_{0}}\right)\right)\right.$ ), at which $F_{3}(\delta)$ and $F_{4}(\delta)$ are respectively greater than $g_{2}(\delta)=$ $4\left(1+\left(\eta^{2}-1\right) /\left(x^{2} \cos ^{2}(3 \pi / 2-\delta)\right)\right)$, defined over $D^{-}$.

Proof: By similar analysis of $F_{3}(\delta), F_{4}(\delta)$, and $g_{2}(\delta)$ over $D^{-}$, we get, $\exists \tilde{\delta}_{31}, \tilde{\delta}_{41} \in D^{-} \ni \forall \delta \in\left(\tilde{\delta}_{31}, \tilde{\delta}_{32}\right), F_{3}(\delta)>$ $g_{2}(\delta)$, and $\forall \delta \in\left(\tilde{\delta}_{41}, \tilde{\delta}_{42}\right), F_{4}(\delta)>g_{2}(\delta)$ where $\tilde{\delta}_{32}=\tilde{\delta}_{42} \triangleq$ $\sup \left\{D^{-}\right\}=\pi-\cos ^{-1}\left(z^{-1} \sin \left(\pi-\theta_{T_{0}}\right)\right)$.

Define $\tilde{\delta}_{i} \triangleq \arg \min \left\{f_{i}(\delta) \mid \delta \in\left[\tilde{\delta}_{i 1}, \tilde{\delta}_{i 2}\right)\right\} \forall i=1,2,3,4$.

Theorem 2: Consider a Retro-PN guided ideal interceptor, pursuing a non-maneuvering target with $\eta \geq 1$. The sufficient conditions for the existence of nontrivial capture zone with constraints on finiteness of LOS turn rate are, $\left(\bar{v}_{0}, \theta_{T_{0}}, \theta_{M_{0}}\right) \in C R_{R P N}$, (given in (32)) and $\beta \in$ $\left[f\left(\tilde{\delta}_{i}\right), f\left(\tilde{\delta}_{i 2}\right)\right)$ when $\left(\bar{v}_{0}, \theta_{T_{0}}, \theta_{M_{0}}\right) \in R_{i}$ for $i=1,2,3,4$.

Proof: By Theorem 1 and the definition of $\underline{\beta}_{1}$, if $\eta=$ $V_{T} / V_{M} \geq 1$ and $\left(\bar{v}_{0}, \theta_{T_{0}}, \theta_{M_{0}}\right) \in R_{1}$ and $\beta \in\left[f_{1}\left(\tilde{\delta}_{1}\right), f_{1}\left(\tilde{\delta}_{12}\right)\right)$, then the retro-PN guided ideal interceptor would intercept the target. From (8)-(10), we obtain,

$$
d w / w=-u w d t=\left(u /\left(u-\beta V_{M}\left(\mathbf{e}_{\mathbf{M}}^{\prime} \mathbf{e}_{\mathbf{r}}\right)\right)\right)(d v / v)
$$

Integrating both sides of (33) in the vicinity of $\theta_{f}$, we get,

$$
\Rightarrow\|\Omega\| \rho^{\left[\left(-2 u_{f}+\beta V_{M}\left(\mathbf{e}_{\mathbf{M}}{ }^{\prime} \mathbf{e}_{\mathbf{r}}\right)_{f}\right) /\left(-u_{f}\right)\right]}=\mathrm{constant}
$$

As $\theta \rightarrow \theta_{f}, u \rightarrow u_{f}<0$ and $v \rightarrow v_{f}=0$ for feasible interception, $\left[-2 u_{f}+\beta V_{M}\left(\mathbf{e}_{\mathbf{M}}^{\prime} \mathbf{e}_{\mathbf{r}}\right)_{f}\right]$ has to be non-positive for finite LOS turn rate. From (22) and its subsequent discussion, $\beta V_{M}\left(\mathbf{e}_{\mathbf{M}}{ }^{\prime} \mathbf{e}_{\mathbf{r}}\right)_{f}-2 u_{f}=\eta^{-1}\left[(\beta+2)\left(\mathbf{e}_{\mathbf{M}}{ }^{\prime} \mathbf{e}_{\mathbf{r}}\right)_{f}+\right.$ $\left.2 \sqrt{\left(\mathbf{e}_{\mathbf{M}} \mathbf{e}_{\mathbf{r}}\right)_{f}^{2}+\left(\eta^{2}-1\right)}\right]$. Since for a retro-PN guided interceptor, $\left(\mathbf{e}_{\mathbf{M}} \mathbf{e}_{\mathbf{r}}\right)_{f}<0$ for feasible interception, as indicated in Lemma 2 ,

$$
\begin{aligned}
& {\left[-2 u_{f}+\beta V_{M}\left(\mathbf{e}_{\mathbf{M}} \mathbf{e}_{\mathbf{r}}\right)_{f}\right] \leq 0 } \\
\Leftrightarrow & (\beta+2)^{2} \geq 4\left(1+\left(\eta^{2}-1\right) /\left(x^{2} \cos ^{2}\left(\theta_{M_{f}}\right)\right)\right)
\end{aligned}
$$

When $\left(\bar{v}_{0}, \theta_{T_{0}}, \theta_{M_{0}}\right) \in R_{1}$ or $R_{2}$, then by Lemma 7 , if $\beta \in\left[f_{1}\left(\tilde{\delta}_{1}\right), f_{1}\left(\tilde{\delta}_{12}\right)\right)$ or $\beta \in\left[f_{2}\left(\tilde{\delta}_{2}\right), f_{2}\left(\tilde{\delta}_{22}\right)\right)$ respectively, then (35) is satisfied achieving feasible interception with finite LOS turn rate. And when $\left(\bar{v}_{0}, \theta_{T_{0}}, \theta_{M_{0}}\right) \in R_{3}$ or $R_{4}$, then by Lemma 8 , if $\beta \in\left[f_{3}\left(\tilde{\delta}_{3}\right), f_{3}\left(\tilde{\delta}_{32}\right)\right)$ or $\beta \in\left[f_{4}\left(\tilde{\delta}_{4}\right), f_{4}\left(\tilde{\delta}_{42}\right)\right)$ respectively, then (35) is satisfied achieving feasible intercep- 


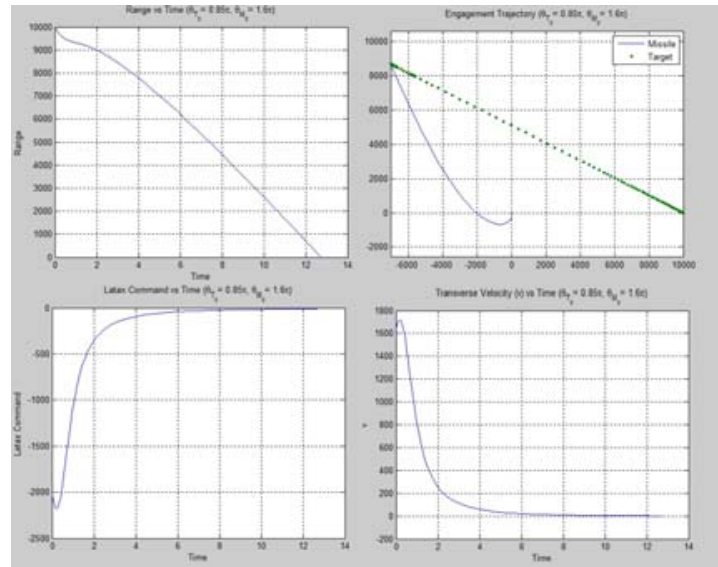

Fig. 5. Engagement Scenario for $\theta_{T_{0}}=0.85 \pi, \theta_{M_{0}}=1.60 \pi$

tion with finite LOS turn rate.

However if $\mathbb{D}_{1}, \mathbb{D}_{2}, \mathbb{D}_{3}, \mathbb{D}_{4}$ are singleton sets, the conditions mentioned in Theorem 2 are also necessary for successful interception with finite LOS turn rate in respective cases.

\section{Simulation Results}

\section{A. Simulation result for planar engagement for $\eta=1.5$}

The simulation result, shown in Fig. 5, is for the engagement parameters $V_{M}=1000 \mathrm{~m} / \mathrm{sec}, V_{T}=1500 \mathrm{~m} / \mathrm{sec}$, $x=1, y=1, \theta_{T_{0}}=0.85 \pi, \theta_{M_{0}}=1.60 \pi$, Initial Range $\left(R_{0}\right)=$ $10000 \mathrm{~m}$. Clearly, $\left(\bar{v}_{0}, \theta_{T_{0}}, \theta_{M_{0}}\right) \in R_{1}$. Applied navigation constant is $-\beta=-f_{1}\left(\tilde{\delta}_{1}\right)=-12.3305$. The variation of $\rho$, $\mathbf{a}_{\mathbf{M}}$ and $v$ with time and the interceptor and target trajectory are shown in Fig. 5. Here $\underline{\delta}_{1}=0.085, \tilde{\delta}_{11}=0.1583=$ $\tilde{\delta}_{1}, \tilde{\delta}_{12}=0.8217$ and $\underline{\beta}_{1}=12.205$. Hence applied $\beta>\underline{\beta}_{1}$ and $\beta=f_{1}\left(\tilde{\delta}_{1}\right) \in\left[f_{1}\left(\tilde{\delta}_{1}\right), f_{1}\left(\tilde{\delta}_{12}\right)\right)$ leading towards successful interception with finite LOS rate.

\section{B. Capture zone of $3 D$ Retro-PN guidance law}

Fig. 6 and 7 show the capture zone of the retro-PN guidance law against high speed non-maneuvering target with $\eta=1.5$ and 1.25 in the $\left(\bar{u}_{0}, \bar{v}_{0}, x\right)$-space for $3 \mathrm{D}$ engagement and in the $\left(\bar{u}_{0}, \bar{v}_{0}\right)$-plane for planar engagement (where $x=y=1$ ) respectively. From Theorem 1, one of the necessary and sufficient conditions for successful interception is $\pi-\sin ^{-1} z<\theta_{T_{0}}<\pi+\sin ^{-1} z$. Clearly by (26), as $x$ increases $\sin ^{-1} z$ also increases resulting into expansion of the capture zone with respect to feasible zone of $\theta_{T_{0}}$, which justifies the pattern of the capture zone in the $\left(\bar{u}_{0}, \bar{v}_{0}, x\right)$ space with respect to $x$. The capture zone is symmetric $\bar{v}_{0}$ and is much smaller $\bar{u}_{0}>0$ than for $\bar{u}_{0}<0$. As $\eta$ increases, the capture zone shrinks since $z$ decreases as $\eta$ increases, as clearly obtained in (26) and validated in Fig. 6.

\section{CONCLUSION}

The conceptualization of retro-PN guidance law has been discussed in this paper. The capturability of this law against high speed non-maneuvering target has been investigated analytically and simulation results have been presented.
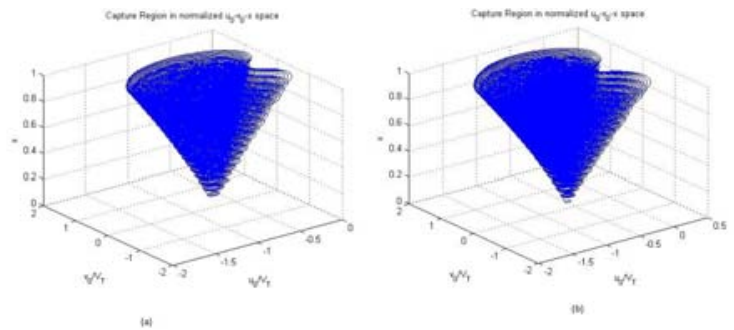

Fig. 6. capture zone of 3D Retro-PN Guidance Law in $\left(\bar{u}_{0}, \bar{v}_{0}, x\right)$-space for $(a) \eta=1.5,(b) \eta=1.25$
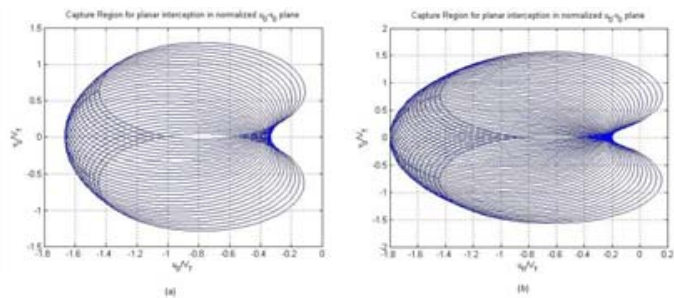

Fig. 7. capture zone of 3D Retro-PN Guidance Law in $\left(\overline{u_{0}}, \overline{v_{0}}\right)$-plane for planar engagement and $(a) \eta=1.5,(b) \eta=1.25$

The simulation results were found to be in line with the theoretical analysis. The capture zone of this law has also been depicted in $\left(\bar{u}_{0}, \bar{v}_{0}, x\right)$-space along with the capture zone for the planar engagement in $\left(\bar{u}_{0}, \bar{v}_{0}\right)$-plane.

\section{REFERENCES}

[1] T. Kuroda and F. Imado, "Advanced Missile Guidance System against Very High Speed Target," in AIAA Guidance, Navigation and Control Conference, Minneapolis, MN, pp. 320-324, 1988.

[2] O. M. Golan and T. Shima, "Head Pursuit Guidance for Hypervelocity Interception", in AIAA Guidance, Navigation, and Control Conference and Exhibit, Providence, RI, Aug 2004, AIAA-2004-4885.

[3] F. Tyan, "Unified Approach to Missile Guidance Laws: A 3D Extension", IEEE Trans. on AES, 41(4), pp. 1178-1199, Oct 2005.

[4] F. Tyan and J.F.Shen, "Capture Region of a Three Dimensional PPN Guidance Law Against a High Speed-Nonmaneuvering Target", in 2008 American Control Conference, Seattle, Washington, pp. 3488-3493, June 2008.

[5] M. Guelman, "A Qualititive Study of Proportional Navigation", IEEE Trans. on AES, AES-7(4), pp. 637-643, July 1971.

[6] M. Guelman, "Proportional Navigation with a Maneuvering Target", IEEE Trans. on AES, 8(3), pp. 364-371, May 1972

[7] S. N. Ghawghawe and D. Ghose, "Pure Proportional Navigation Against Time-Varying Target Maneuvers", IEEE Trans. on AES, 32(4), pp. 1336-1347, Oct 1996.

[8] H. M. Prasanna and D. Ghose, "Capturability of Modified Proportional Naigation for Intercepting High Speed Targets", Technical Report GCDSL/2006/02, Guidance, Control, and Decision Systems Laboratory, Department of Aerospace Engineering, Indian Institute of Science, Bangalore, May 2006.

[9] H. M. Prasanna and D. Ghose, "A Generalized Guidance Law for Intercepting High Speed Targets", Proceedings of the International Conference on Advances in Control and Optimization of Dynamical Systems (ACODS2007), pp. 211-217, Jan 2007.

[10] H.M. Prasanna and D. Ghose, "Retro-PN: A new guidance law for interception of high speed targets", Journal of guidance, Control, and Dynamics (to appear).

[11] F. Tyan, "Capture Region of a GIPN Guidance Law for Missile and Target with Bounded Maneuverability", IEEE Trans. on AES, 47(1), pp. 201-213, Jan 2011.

[12] E. Duflos, P. Penel and P. Vanheeghe, "3D guidance law modeling", IEEE Trans. on AES, 35(1), pp. 72-83, Jan 1999. 